\title{
Color Image Analysis of Cosmetic Web-Site for Color Marketing
}

\author{
Lee Jeongman ${ }^{\star} \cdot$ An Jongsuk ${ }^{* *}$ \\ Professor, DASAN LINC Educational Development Institute, Dankook University* \\ Professor, Beauty Design, Venture Graduate School of Hoseo University ${ }^{\star \star}$
}

\begin{abstract}
This study aims to derive the sensitive image analysing the color tones based on the web-sites of cosmetic brand and to know how the web-site color tones and the color tone image as visual factor for information transfer do influence the effective communication. 10 cosmetic brand web-sites are selected based on the pre survey, and the main page color tones of cosmetic brand web-site are analyzed by Color Catch and Munsell Conversion program, whereas the composition of color tones and color image such as the main trend colors, secondary colors, accent colors and etc are analyzed by a graphic professional program 'Adobe Photoshop'. 5 color tone groups are classified as Black\&White, Green\&White, Blue\&White, Purple\&White and Red\&White according to the color distribution chart, and the emotional language is derived applying them to the pre research and $\mid \mathrm{RI}$ image scale. The color association is empirically analyzed by a survey method. The study results finally show Black\&White is proper for expression of high quality and modern image, Green\&White for pure and clean image, Blue\&White for cool and transparent image, Purple\&White for mysterious and elegant image respectively. This study results are necessary for plan of color delivering the strong transferring power of visual information the time when to make the effective web-sites, and it hopefully will be utilized as the basic data for the color marketing to actively express the brand identity and to satisfy the consumers' sensitivity.
\end{abstract}

Key Words : Color Tone, Color Tone Image, Color Marketing, Cosmetic Web-Site

\section{Introduction}

\section{Necessity of study}

The current market is changed to the shape of preferable consumption based on sensitivity and taste. Y.C. Liu(2010) told, the visual factor is the most influential factor for delivery of brand image to web user." ${ }^{1)}$ The color tone is the strong

Corresponding author: An Jongsuk, Tel. +82-41-560-8436, Fax. +82-41-560-8436

E-mail: modean@hoseo.edu. 
transfer means to recognize the object most quickly for the human beings who get the most information by sight. The color tone is able to largely influence the recognition and trust of brand, and so the color marketing is intended to increase the product image and preference, or to differentiate the brand and to advertise the product. The digital networks in 21 centuries are very quickly developed as nobody expects, and the competition to get the trust from consumer is getting harder utilizing the approach easy web-sites that have no limit of time and space. Therefore, the study on the color application that have the strong transfer power of visual information is needed for the production of the effective web-site.

This study aims to investigate the influence of web-site color and color image as a visual factor of information transfer on the effective communication based on the high value cosmetic brand as a new growing industry.

The study issues are as follows.

First, the color images will be derived analyzing the web-site color tone of cosmetic brand.

Second, it will be investigated what kind of emotional language will be the web-site color tone delivered by.

The results of this study hopefully will be utilized for the basic data of color marketing to actively express the brand identity and fulfil the consumer sensitivity.

\section{Methods and scope of study}

The consumer preference is pre surveyed for brands available at department store of which cosmetic market share and consumer taste are high. 100 male and female are interviewed who purchased the cosmetics at Choongchung Galleria department store on 2nd April 2010.
Each main page of the most aware 10 brand web-sites according to the survey is selected as basic data for analysis(Table 3). The colors are extracted for color analysis using the digital colors according to characters of web-site colors namely, HTML color code, whereas the color information and values are searched substituting HV/C by Color Cache \& Munsell Conversion(ver.10.0.8a). The tone images are derived finding the color position by substituting the analyzed colors in chart of KS A 0011 (name of object color), three attribute of color display. The web-site main pages are pixel transformed by the graphic professional program 'Adobe Photoshop' and the dominant colors, assort colors and accent colors are classified analyzing the color distribution area. IRI Image Scale is used for the image analysis of web page's color scheme. In order to investigate what image delivered the brand color, the color images are classified as 5 groups by color analysis of cosmetic web-site, and then the main survey is completed. The survey is carried out in form of personal interview for 20 days from 10th June 2010, and totally 325 questionnaires are collected on Seoul station and Cheonan bus terminal.

\section{Theoretical background}

\section{Color tone and color association}

According to the color definition of Y.S. Park(2007), the color as a physical phenomenon is recognized through the sensory organ 'eye' or it is the experience effects like recognitive phenomenon. The color is also referred to the physical object 'light' as visual recognitive object and so the recognitive phenomenon of the light. The color tone is recognized as the color of a 
physical phenomenon through the sensory organ 'eye' or it is referred to the experience effects like recognitive phenomenon. Therefore, the color is referred to a physical phenomenon, whereas the color tone is referred to a psychological phenomenon. ${ }^{2)}$

The color tone does play the important role continuously for transferring the social cultural visual message. The enterprise has utilized the influential power of color tone since establishing the definition of brand identity. Truly Deeply(2010) told that the color tone is sensitively reactive to the trend and so the colors are utilized independent on the established meaning of the brand due to the color preference, and sometimes it transfers the mixed messages. ${ }^{3)}$

The color association is to connect with the activity or state according to the experience and psychological action when we recognize the color. The certain object or feeling is associated when we recognize the color. However, the color association is ruled by the personal characters, remembrance and experience and different according to the life style, culture, location, environment sexuality, age and etc. And if the majority share the sympathy, then the color will be the symbol with the social characters.
$<$ Table 1> shows the color association based on the overlapped words referred to the pre search of S.H. Park, H.J. Jeong(2010) and J.H. $\operatorname{Kim}(2007) .{ }^{4), 5)}$

\section{Digital color}

Digital screen is composed of pixel, and it's color is expressed using electric line, each pixel is the color combined with RGB of the three primary colors, and a pixel involves a lot of information. The number of color is indicated according to the number of bit as table. ${ }^{6)}$

RGB expressed by decimal scale should be changed to by hexadecimal number system. If we transfer from 0 to 255, then FF from 00 becomes the color code of HTML. The code 000000 among the HTML codes becomes the black color with no light, and the reverse FFFFFF becomes the white color with the richest light.

\section{Color marketing}

Color marketing is to manage the colors in order to increase the image and preference of product or to differentiate or advertise it. ${ }^{7)}$ In other words, the color marketing is a color policy

$<$ Table $1>$ Color association

\begin{tabular}{l|l}
\hline \hline \multicolumn{1}{c|}{ Color } & \multicolumn{1}{c}{ Association \& Symbol } \\
\hline Red(R) & stimulating, passion, exciting, affection, danger, voluptuous \\
\hline Yellow(Y) & joy, hope, pleasure, pleasant, light \\
\hline Green(G) & peace, refreshing, hope, rest, stability, calm, freshness \\
\hline Blue(B) & young, cool, meditation, clearness, transparent, cold \\
\hline Purple(P) & creation, loneliness, mystery, arts, dignity, provocative, brilliant, elegant \\
\hline White(W) & purity, virginity, sacred, honesty, simple, clean \\
\hline Gray $(\mathrm{N})$ & common, modesty, sorghum, gloomy, lethargy, urban \\
\hline Black(K) & creation, luxury, mystery, elegancy, dignity, polished \\
\hline \hline
\end{tabular}


<Table 2> Digital color system

\begin{tabular}{c|r|l}
\hline \hline bit & Color & Remarks \\
\hline 1 & 2 & White\&Black \\
\hline 2 & 4 & Palette \\
\hline 4 & 16 & Palette \\
\hline 8 & 256 & Palette \\
\hline 16 & 65,536 & High Color \\
\hline 24 & $16,777,216$ & True Color \\
\hline 32 & $16,777,216+8$ bit achannel & True Color \\
\hline \hline
\end{tabular}

for display of interest in the relevant product by visual expression of the colors consumers yearn for. Color marketing provide with the product specifications and information, and so the consumption will be promoted by inducement of the images preferred. $^{8)}$

The various senses will make big influence on decision when to choose the product. Especially, the visual independence is over 10 times higher than other hearing and tactile. The fixing consumer's eye on a product is lower than 0.1 second and so the color's influence will be getting bigger.

Color marketing is the sales strategy to maximize the sale using the colors. The product plan of the enterprise should be related to the consumer and the color maturity should be high according to the consumer's sensitivity. The color marketing strategy should be a circulative coordination plan like 'Plan-Production-SalePlan' and the consumer's psychology as 'Person -Heart-Color-Product' should be also considered in product. $^{9)}$

The color among designs is a decisive factor for product since the equalization of enterprises manufacturing technologies, and human beings show the emotional reaction to colors, and this is directly connected to purchase impulse. That is basic logics of marketing.

\section{Color image of cosmetic web-site}

\section{Color analysis of cosmetic web-site}

$<$ Table 3> shows the 10 highest awareness firms of cosmetic brand web-site according to the pre survey. The main home page colors of the selected cosmetic brands are analyzed by Adobe Photoshop, Color Cache and Munsell Conversion, and they are also analyzed based on associated images of pre studies. According to the distribution chart, about $60 \sim 70 \%$ area is classified as dominant color, $20 \sim 30 \%$ of the total area is assort color supporting the dominant color, and the strong color tone, accent color, is also classified showing the change of total style and making the fresh effects. These classifications is able to deliver the clear color arrangement. ${ }^{10)}$

For the color analysis, all colors of the web-site main page are processed by Adobe Photoshop in pixel, the colors with very low differences of $\mathrm{HV} / \mathrm{C}$ value are primarily bound as the similar color, and the colors with below than $0.10 \%$ of distribution secondarily are classified as etc.

\section{1) Analysis of CHANEL color}

The CHANEL main web-site page as <Fig. 1> is divided in width 111 and height 72 with total 7,992 pixels by Adobe Photoshop, and the pixel transformed main page colors are totally 241 . The similar colors showing the very low difference of $\mathrm{HV} / \mathrm{C}$ value are primarily classified in 30 colors by color cache, and these also are classified as dominant color, assort color and accent color according to the distribution chart. The derived color combination as <Table 5> shows that the tone is composed of dominant 
$<$ Table 3> Cosmetic brands selected

\begin{tabular}{l|l}
\hline \hline \multicolumn{1}{c|}{ Brand } & \multicolumn{1}{c}{ Web-Site } \\
\hline CHANEL & http://www.chanel.com/ \\
\hline benefit & http://www.benefitkorea.co.kr/ \\
\hline CLINIQUE & http://www.cliniquekorea.co.kr/ \\
\hline HERA & http://www.hera.co.kr/ \\
\hline Dior & http://www.dior.com/ \\
\hline IOPE & http://www.iope.co.kr/ \\
\hline ESTEE LAUDER & http://www.esteelauder.co.kr/ \\
\hline LANCOME & http://www.lancome.co.kr/ \\
\hline O HUI & http://www.ohui.co.kr/ \\
\hline SK-II & http://www.sk2.co.kr/ \\
\hline \hline
\end{tabular}

※ Standard as May 2010

colors such as chromatic of black PB, white GY and Iow chroma BG affiliation maximizing the brightness differences, and BG shown by CHANEL as light grayish tone delivering the light, thin, soft image of achromatic color is maximized.

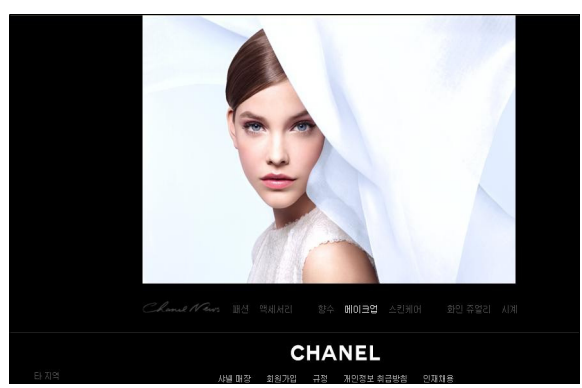

<Fig. 1> CHANEL Homepage

2) Analysis of benefit Color

The benefit main web-site page as <Fig. 2> is divided in width 111 and height 71 with total 7,952 pixels by Adobe Photoshop and the pixel
<Table 4> CHANEL Color distribution chart

\begin{tabular}{c|c|r}
\hline \hline color & HTML Hex RGB & \multicolumn{1}{c}{ Ratio } \\
\hline & 000000 & $47.07 \%$ \\
\hline & ffffff & $34.32 \%$ \\
\hline & ccffff & $3.00 \%$ \\
\hline & cccccc & $2.77 \%$ \\
\hline & ccccff & $2.46 \%$ \\
\hline & 666666 & $2.03 \%$ \\
\hline & 333333 & $1.88 \%$ \\
\hline & ffcccc & $1.83 \%$ \\
\hline & cc9999 & $1.04 \%$ \\
\hline & 999999 & $0.89 \%$ \\
\hline & 996666 & $0.71 \%$ \\
\hline & 663333 & $0.58 \%$ \\
\hline & ffcc99 & $0.25 \%$ \\
\hline & 330000 & $0.20 \%$ \\
\hline & ffffcc & $0.16 \%$ \\
\hline & ffccff & $0.14 \%$ \\
\hline & 666633 & $0.13 \%$ \\
\hline etc. & cc9966 & $0.10 \%$ \\
\hline \hline & 12 color & $0.46 \%$ \\
\hline & 30 color & $100.00 \%$ \\
\hline
\end{tabular}

$<$ Table 5> Main color scheme of CHANEL

\begin{tabular}{|c|c|c|}
\hline \multirow{9}{*}{$\begin{array}{c}\text { Dominant } \\
\text { Color }\end{array}$} & $\mathrm{Hex}$ & $\# 000000$ \\
\hline & Hue & 6.62PB 1.06/15.42 \\
\hline & RGB & $(0,0,0)$ \\
\hline & Hex & \#fdfdff \\
\hline & Hue & 9.39GY 9.83/0.69 \\
\hline & RGB & $(253.253 .255)$ \\
\hline & Hex & $\# \mathrm{e} 7 \mathrm{f} 4 \mathrm{fd}$ \\
\hline & Hue & 6.54BG 9.4/1.37 \\
\hline & RGB & $(231,244,253)$ \\
\hline
\end{tabular}

transformed main page colors are totally 253. The similar colors showing the very low difference of $\mathrm{HV} / \mathrm{C}$ value are primarily classified in 62 colors by color cache. Classifying the dominant color, assort color and accent color 


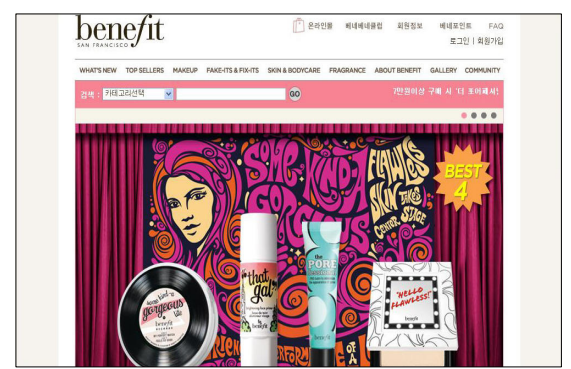

<Fig. 2> benefit Homepage

$\langle$ Table 6$\rangle$ benefit color distribution chart

\begin{tabular}{|c|c|c|}
\hline color & HTML Hex RGB & 비율 \\
\hline & ffffff & $37.69 \%$ \\
\hline & 660033 & $7.38 \%$ \\
\hline & 000000 & $5.41 \%$ \\
\hline & 993366 & $5.17 \%$ \\
\hline & $\operatorname{cccccc}$ & $5.07 \%$ \\
\hline & 333333 & $3.28 \%$ \\
\hline & 993333 & $2.94 \%$ \\
\hline & $\mathrm{ffcccc}$ & $2.25 \%$ \\
\hline & 663366 & $2.24 \%$ \\
\hline & ff9999 & $2.05 \%$ \\
\hline & 999999 & $1.82 \%$ \\
\hline & ff6699 & $1.70 \%$ \\
\hline & 663333 & $1.70 \%$ \\
\hline & 666666 & $1.65 \%$ \\
\hline & cc3399 & $1.56 \%$ \\
\hline & 990033 & $1.16 \%$ \\
\hline & 996666 & $1.08 \%$ \\
\hline & ccffff & $1.07 \%$ \\
\hline & 000033 & $1.03 \%$ \\
\hline & 330033 & $0.87 \%$ \\
\hline & 999966 & $0.84 \%$ \\
\hline & cc6633 & $0.79 \%$ \\
\hline & 996699 & $0.78 \%$ \\
\hline & 330000 & $0.72 \%$ \\
\hline & cc3366 & $0.70 \%$ \\
\hline & cc9966 & $0.60 \%$ \\
\hline & 99сссс & $0.59 \%$ \\
\hline & 003333 & $0.57 \%$ \\
\hline & ffcc99 & $0.50 \%$ \\
\hline & 63366 & $0.45 \%$ \\
\hline & ff99cc & $0.43 \%$ \\
\hline & ffffcc & $0.40 \%$ \\
\hline & 66633 & $0.31 \%$ \\
\hline & ff9933 & $0.29 \%$ \\
\hline & $\operatorname{cccc} 99$ & $0.28 \%$ \\
\hline & 990066 & $0.28 \%$ \\
\hline & 669999 & $0.28 \%$ \\
\hline & 993399 & $0.18 \%$ \\
\hline etc. & 24 color & $3.90 \%$ \\
\hline Total & 62 color & $100.00 \%$ \\
\hline
\end{tabular}

according to the distribution chart, the derived color combination is as $\langle$ Table 7$\rangle$. For color tone, the vivid tone $\mathrm{R}$ and RP affiliation(45\%) and achromatic White(37\%) are used as dominant color, and so the splendid, strong, proactive, stimulating, active image and bold expression are effectively delivered. Using BG and YR of deep tone as assort color, the deep, traditional, courteous, loyal, exclusive and classical image is well expressed.

$<$ Table 7> Main color arrangement of benefit

\begin{tabular}{|c|c|c|}
\hline \multirow{12}{*}{$\begin{array}{c}\text { dominant } \\
\text { color }\end{array}$} & Hex & \#f88393 \\
\hline & Hue & $2.52 R \quad 6.42 / 11.14$ \\
\hline & RGB & $(248,131,147)$ \\
\hline & Hex & \#b62561 \\
\hline & Hue & 0.09R 3.74/12.89 \\
\hline & RGB & $(182,37,97)$ \\
\hline & Hex & \#d32589 \\
\hline & Hue & 6.03RP 4.47/16.13 \\
\hline & RGB & $(211,37,137)$ \\
\hline & Hex & \#ffffff \\
\hline & Hue & 8.48GY 9.9/0.78 \\
\hline & RGB & $(255,255,255)$ \\
\hline \multirow{6}{*}{$\begin{array}{l}\text { accent } \\
\text { color }\end{array}$} & Hex & \#191d2d \\
\hline & Hue & 6.63BG 9.01/9.93 \\
\hline & RGB & $(25,29,45)$ \\
\hline & Hex & \#cd6716 \\
\hline & Hue & 2.87YR 5/12.49 \\
\hline & RGB & $(205,103,22)$ \\
\hline
\end{tabular}

3) Analysis of CLINIQUE color

The CLINIQUE web-site main page as <Fig. $3>$ is divided in width 115 and height 73 with total 8,395 by Adobe Photoshop and the pixel 
transformed main page colors are totally 242. The similar colors showing the very low difference of $\mathrm{HV} / \mathrm{C}$ value are primarily classified in 26 colors by color cache. Classifying the dominant color, assort color and accent color according to the distribution chart, the derived color combination is as 〈Table 9>. Black is used as accent color, whereas White GY (68\%) of achromatic color is used as dominant color and $G$ affiliation of pale tone is used as assort color. This color arrangement expresses the light, soft, weak, female image and the luxurious color effects.

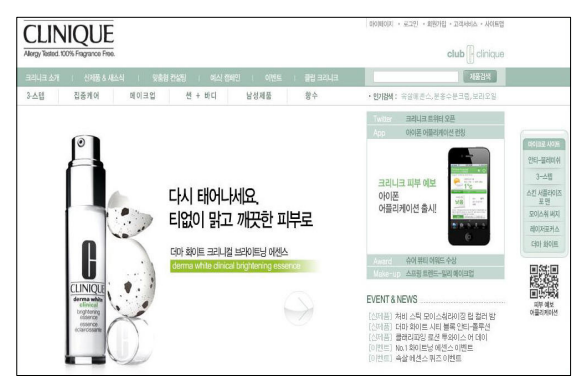

<Fig. 3> CLINIQUE Homepage

<Table 8> CLINIQUE color distribution chart

\begin{tabular}{c|c|r}
\hline \hline color & HTML Hex RGB & Ratio \\
\hline & fffff & $68.47 \%$ \\
\hline & cccccc & $12.52 \%$ \\
\hline & 000000 & $4.74 \%$ \\
\hline & 999999 & $3.56 \%$ \\
\hline & ccffcc & $2.98 \%$ \\
\hline & $99 c c c c$ & $2.17 \%$ \\
\hline & 666666 & $1.00 \%$ \\
\hline & 99 cc99 & $0.96 \%$ \\
\hline & ccffff & $0.95 \%$ \\
\hline & 333333 & $0.50 \%$ \\
\hline & cccc66 & $0.44 \%$ \\
\hline & $9999 c c$ & $0.33 \%$ \\
\hline & ffffcc & $0.31 \%$ \\
\hline & cc99cc & $0.29 \%$ \\
\hline & $99 c c 66$ & $0.23 \%$ \\
\hline Total & cccc99 & $0.23 \%$ \\
\hline \hline & & $0.32 \%$ \\
\hline
\end{tabular}

<Table 9> Main color arrangement of CLINIQUE

\begin{tabular}{l|l|l|l|}
\hline \hline \multirow{2}{*}{$\begin{array}{c}\text { dominant } \\
\text { color }\end{array}$} & & & \\
\hline
\end{tabular}

4) Analysis of HERA color

The HERA web-site main page as <Fig. $4>$ is divided in width 119 and height 73 with total 8,687 by Adobe Photoshop and the pixel transformed main page colors are totally 256. The similar colors showing the very low difference of $\mathrm{HV} / \mathrm{C}$ value are primarily classified in 37 colors by color cache. Classifying the dominant color, assort color and accent color according to the distribution chart, the derived color combination is as <Table 11>. BG and GY(59\%) are used as dominant color and $P$ affiliation(18\%) of low chroma is used as assort color with the color scheme showing the brightness difference. The light, thin, soft and elegant image is expressed using BG, the light grayish tone of high brightness and GY as dominant color(59\%), whereas the deep, traditional, courteous, luxurious and classical image is expressed using $P$ affiliation of deep tone as assort color(18\%). 


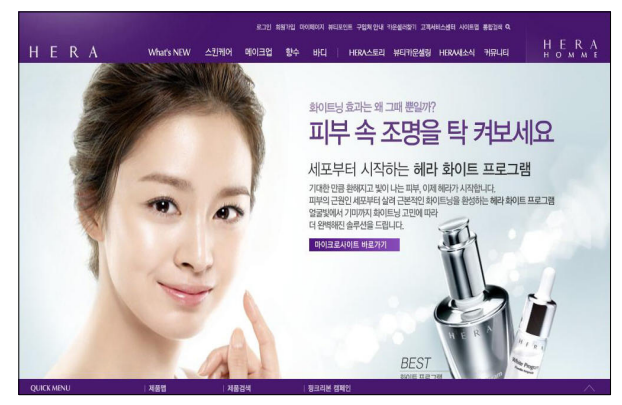

<Fig. 4> HERA Homepage

<Table 10> HERA color distribution chart

\begin{tabular}{|c|c|c}
\hline \hline color & HTML Hex RGB & Ratio \\
\hline & fffff & $30.84 \%$ \\
\hline & cccccc & $12.84 \%$ \\
\hline & ccffff & $7.84 \%$ \\
\hline & ffcccc & $5.92 \%$ \\
\hline & 000000 & $5.03 \%$ \\
\hline & 330066 & $4.47 \%$ \\
\hline & cccc99 & $3.58 \%$ \\
\hline & 663366 & $3.32 \%$ \\
\hline & ccccff & $3.02 \%$ \\
\hline & 333333 & $1.98 \%$ \\
\hline & 999999 & $1.97 \%$ \\
\hline & cc9999 & $1.97 \%$ \\
\hline & 666699 & $1.95 \%$ \\
\hline & 663399 & $1.72 \%$ \\
\hline & 333366 & $1.58 \%$ \\
\hline & 996699 & $1.57 \%$ \\
\hline & $9999 \mathrm{cc}$ & $1.24 \%$ \\
\hline & ffffcc & $0.94 \%$ \\
\hline & 663333 & $0.84 \%$ \\
\hline & 666666 & $0.76 \%$ \\
\hline & 996666 & $0.71 \%$ \\
\hline ccffcc & $0.69 \%$ \\
\hline Total & cc99cc & $0.51 \%$ \\
\hline & ffcc99 & $0.46 \%$ \\
\hline & cc6666 & $0.10 \%$ \\
\hline & 12 color & $4.16 \%$ \\
\hline & 37 color & $100.00 \%$ \\
\hline & &
\end{tabular}

5) Analysis of Dior color

The Dior web-site main page as 〈Fig. 5$\rangle$ is divided in width 119 and height 77 with total 8,687 by Adobe Photoshop and the pixel transformed main page colors are totally 255. The similar colors showing the very low difference of $\mathrm{HV} / \mathrm{C}$ value are primarily classified in 40 colors by color cache. Classifying the dominant color, assort color and accent color according to the distribution chart, the derived color combination is as <Table 13>. The clean, polished and modern image is expressed using Black \& White affiliation as dominant color(81\%), whereas the light, thin, elegant and polished image is expressed using BG of light grayish as assort color. The health, female, fresh and bright image is expressed using $Y$ of bright tone as accent color.

$<$ Table 11> Main color arrangement of HERA
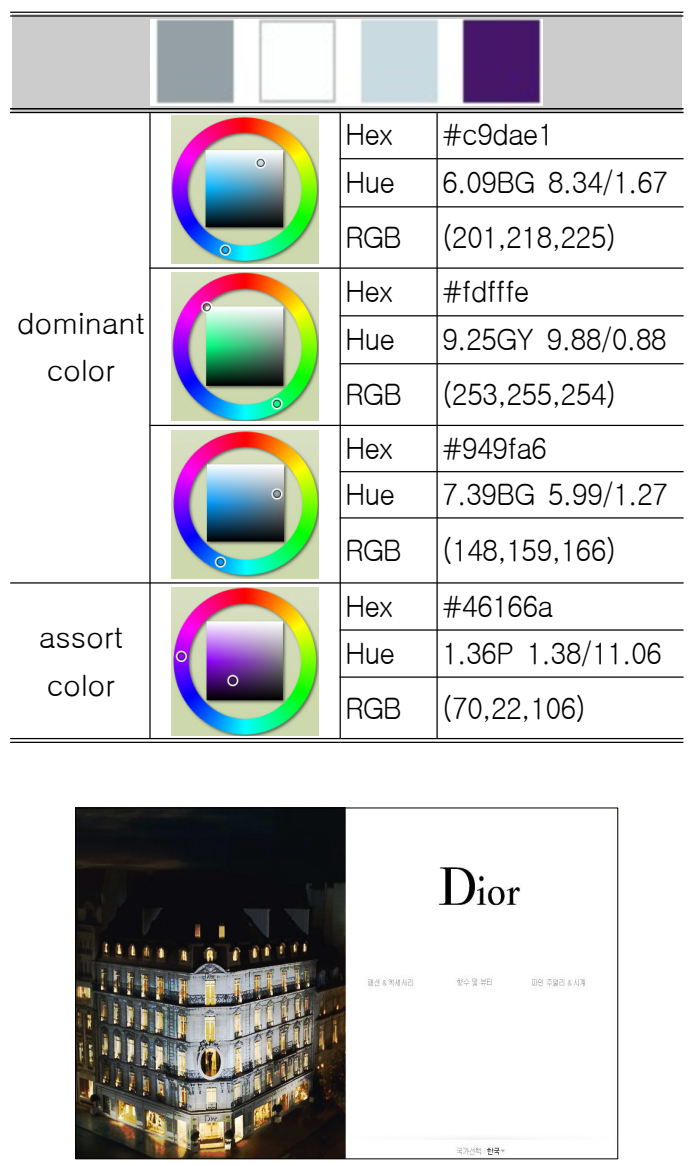

Dior

<Fig. 5> Dior Homepage 
<Table 12> Dior color distribution chart

\begin{tabular}{|c|c|c|}
\hline color & HTML Hex RGB & Ratio \\
\hline & ffffff & $48.49 \%$ \\
\hline & 000000 & $26.21 \%$ \\
\hline & 666666 & $4.53 \%$ \\
\hline & 333333 & $3.97 \%$ \\
\hline & 999999 & $2.42 \%$ \\
\hline & $\operatorname{cccccc}$ & $1.84 \%$ \\
\hline & 666633 & $1.55 \%$ \\
\hline & 333300 & $1.54 \%$ \\
\hline & 999966 & $1.32 \%$ \\
\hline & 663333 & $0.97 \%$ \\
\hline & 996666 & $0.58 \%$ \\
\hline & 996633 & $0.56 \%$ \\
\hline & $\operatorname{cccc} 99$ & $0.56 \%$ \\
\hline & 330000 & $0.53 \%$ \\
\hline & $99 \mathrm{cccc}$ & $0.48 \%$ \\
\hline & cc9966 & $0.46 \%$ \\
\hline & 003300 & $0.40 \%$ \\
\hline & cc9999 & $0.35 \%$ \\
\hline & 003333 & $0.35 \%$ \\
\hline & 9999cc & $0.33 \%$ \\
\hline & 666699 & $0.32 \%$ \\
\hline & 669999 & $0.31 \%$ \\
\hline & 663300 & $0.29 \%$ \\
\hline & cccc66 & $0.19 \%$ \\
\hline & ffff99 & $0.19 \%$ \\
\hline & ffcc99 & $0.17 \%$ \\
\hline & 336666 & $0.16 \%$ \\
\hline & ffffcc & $0.16 \%$ \\
\hline & 000033 & $0.15 \%$ \\
\hline & ffcc66 & $0.14 \%$ \\
\hline & cc9933 & $0.11 \%$ \\
\hline etc. & 9 color & $0.35 \%$ \\
\hline Total & 40 color & $100.00 \%$ \\
\hline
\end{tabular}

6) Analysis of IOPE color

The IOPE web-site main page as <Fig. 6> is divided in width 82 and height 51 with total 4,128 by Adobe Photoshop and the pixel transformed main page colors are totally 253. The similar colors showing the very low difference of $\mathrm{HV} / \mathrm{C}$ value are primarily classified in 38 colors by color cache. Classifying the dominant color, assort color and accent color according to the distribution chart, the derived $\langle$ Table 13> Main color arrangement of Dior

\begin{tabular}{l|l|l|l}
\hline \hline & & & \\
\hline
\end{tabular}

color combination is as <Table 15>. The health, female, fresh image and splendid feeling are expressed using $G$ affiliation of achromatic color and $B G$ of bright tone, whereas the deep, traditional, courteous, faithful, luxurious and classical image is expressed and dominant color (62\%) arranges the colors with difference of chroma and brightness.

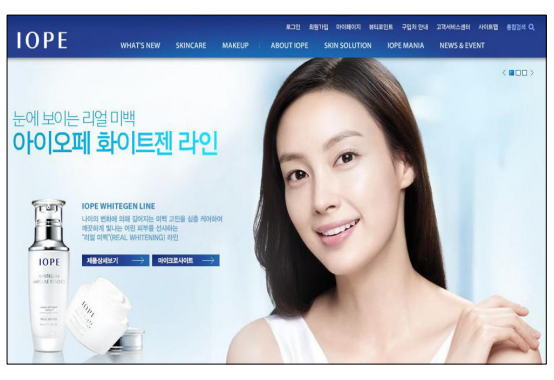

<Fig. 6> IOPE Homepage 
$<$ Table 14> IOPE color distribution chart

\begin{tabular}{l|c|r}
\hline \hline color & HTML Hex RGB & Ratio \\
\hline & ffffff & $26.64 \%$ \\
\hline & ccffff & $19.89 \%$ \\
\hline & 000000 & $8.01 \%$ \\
\hline & ffcccc & $7.96 \%$ \\
\hline & ccccff & $5.69 \%$ \\
\hline & cccccc & $4.02 \%$ \\
\hline & 333399 & $3.83 \%$ \\
\hline & 333333 & $3.13 \%$ \\
\hline & $6699 \mathrm{cc}$ & $2.56 \%$ \\
\hline & cc9999 & $2.46 \%$ \\
\hline & cccc99 & $2.10 \%$ \\
\hline & 336699 & $2.06 \%$ \\
\hline & $9999 \mathrm{cc}$ & $2.01 \%$ \\
\hline & 330000 & $1.10 \%$ \\
\hline & 666699 & $0.91 \%$ \\
\hline & 666666 & $0.91 \%$ \\
\hline & 996666 & $0.72 \%$ \\
\hline & 999999 & $0.69 \%$ \\
\hline & $99 \mathrm{ccff}$ & $0.67 \%$ \\
\hline & $99 \mathrm{cccc}$ & $0.62 \%$ \\
\hline & 333300 & $0.50 \%$ \\
\hline etc. & $3366 \mathrm{cc}$ & $0.45 \%$ \\
\hline & 663333 & $0.45 \%$ \\
\hline & 999966 & $0.41 \%$ \\
\hline & $939 f f f f$ & $0.38 \%$ \\
\hline & 336666 & $0.10 \%$ \\
\hline & 38 color & $1.72 \%$ \\
\hline & & $100.00 \%$ \\
\hline & &
\end{tabular}

$<$ Table 15> Main color arrangement of IOPE

\begin{tabular}{|c|c|c|}
\hline \multirow{12}{*}{$\begin{array}{c}\text { dominant } \\
\text { color }\end{array}$} & Hex & \#fbfcfe \\
\hline & Hue & 0.04G 9.79/0.72 \\
\hline & RGB & $(251,252,254)$ \\
\hline & $\mathrm{Hex}$ & $\# 1 \mathrm{f4} 190$ \\
\hline & Hue & 6.48PB 2.37/11.04 \\
\hline & RGB & $(31,65,144)$ \\
\hline & Hex & \#c0dde9 \\
\hline & Hue & 3.8BG 8.36/2.49 \\
\hline & RGB & $(192,221,223)$ \\
\hline & $\mathrm{Hex}$ & \#00dafb \\
\hline & Hue & 3B 7.78/9.3 \\
\hline & RGB & $(0,218,251)$ \\
\hline
\end{tabular}

\section{7) Analysis of ESTEE LAUDER color}

The ESTEE LAUDER web-site main page as <Fig. 7> is divided in width 89 and height 55 with total 4,895 by Adobe Photoshop and the pixel transformed main page colors are totally 250. The similar colors showing the very low difference of $\mathrm{HV} / \mathrm{C}$ value are primarily classified in 33 colors by color cache. Classifying the dominant color, assort color and accent color according to the distribution chart, the derived color combination is as <Table 17>. The light, soft, weak and female image is expressed using $G$ affiliation of achromatic color and BG of pale tone as dominant color(52\%), whereas the deep, traditional, courteous, faithful, luxurious and classical image is expressed using PB of deep tone.

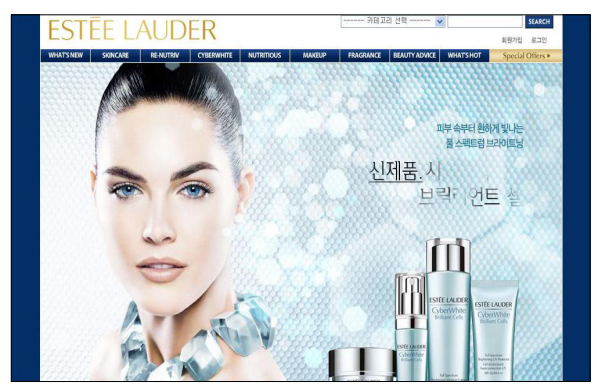

<Fig. 7> ESTEE LAUDER Homepage

8) Analysis of LANCOME color

The LANCOME web-site main page as <Fig. $8>$ is divided in width 94 and height 59 with total 5,546 by Adobe Photoshop and the pixel transformed main page colors are totally 249. The similar colors showing the very low difference of $\mathrm{HV} / \mathrm{C}$ value are primarily classified in 33 colors by color cache. Classifying the dominant color, assort color and accent color according to the distribution chart, the derived color combination is as <Table 19>. The light, 
thin, soft, elegant and polished image is expressed using White(GY 53\%) of achromatic

$<$ Table 16> ESTEE LAUDER color distribution chart

\begin{tabular}{|c|c|c|}
\hline \multirow[t]{27}{*}{ color } & HTML Hex RGB & Ratio \\
\hline & ffffff & $30.42 \%$ \\
\hline & $\operatorname{ccccc} C$ & $12.63 \%$ \\
\hline & ccffff & $10.68 \%$ \\
\hline & ccccff & $10.44 \%$ \\
\hline & $\mathrm{ffcccc}$ & $6.31 \%$ \\
\hline & 000000 & $6.29 \%$ \\
\hline & 003366 & $4.33 \%$ \\
\hline & 99 cссс & $3.88 \%$ \\
\hline & 669999 & $2.47 \%$ \\
\hline & 999999 & $2.47 \%$ \\
\hline & 336666 & $1.74 \%$ \\
\hline & 9999сc & $1.61 \%$ \\
\hline & cc9999 & $0.96 \%$ \\
\hline & ffffcc & $0.84 \%$ \\
\hline & 336699 & $0.82 \%$ \\
\hline & 333333 & $0.82 \%$ \\
\hline & 666666 & $0.65 \%$ \\
\hline & $\operatorname{cccc99}$ & $0.55 \%$ \\
\hline & 333366 & $0.53 \%$ \\
\hline & 996666 & $0.43 \%$ \\
\hline & cc9966 & $0.18 \%$ \\
\hline & 000066 & $0.14 \%$ \\
\hline & 000033 & $0.12 \%$ \\
\hline & 666633 & $0.10 \%$ \\
\hline & 666699 & $0.10 \%$ \\
\hline & ffcc99 & $0.10 \%$ \\
\hline etc. & 7 color & $0.36 \%$ \\
\hline Total & 33 color & $100.00 \%$ \\
\hline
\end{tabular}

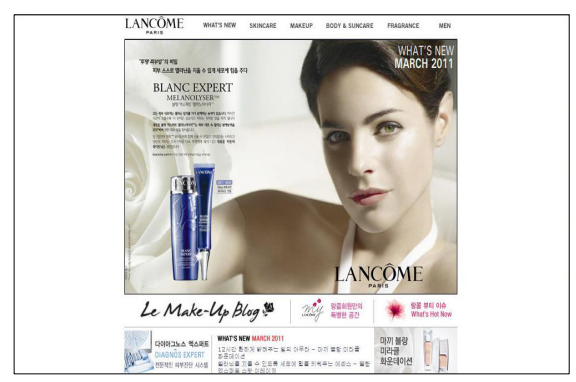

<Fig. 8> LANCOME Homepage
$<$ Table 17> Main color arrangement of ESTEE LAUDER

\begin{tabular}{|c|c|c|}
\hline \multirow{9}{*}{$\begin{array}{c}\text { dominant } \\
\text { color }\end{array}$} & Hex & \#fafbff \\
\hline & Hue & 1.59G 9.75/0.63 \\
\hline & RGB & $(250,251,255)$ \\
\hline & Hex & \#c6dee8 \\
\hline & Hue & $9.12 B G \quad 8.46 / 2.24$ \\
\hline & RGB & $(198,222,232)$ \\
\hline & Hex & $\# c 1 d 6 d b$ \\
\hline & Hue & 5.08BG 8.14/1.93 \\
\hline & RGB & $(193,214,219)$ \\
\hline \multirow{3}{*}{$\begin{array}{l}\text { assort } \\
\text { color }\end{array}$} & Hex & $\# 042 f 66$ \\
\hline & Hue & 5.98PB 1.36/8.06 \\
\hline & RGB & $(4,47,102)$ \\
\hline
\end{tabular}

<Table 18> LANCOME color distribution chart

\begin{tabular}{c|c|r}
\hline \hline color & HTML Hex RGB & \multicolumn{1}{c}{ Ratio } \\
\hline & fffff & $53.21 \%$ \\
\hline & cccccc & $16.91 \%$ \\
\hline & 000000 & $5.34 \%$ \\
\hline & 666633 & $3.25 \%$ \\
\hline & cccc99 & $3.14 \%$ \\
\hline & 999999 & $2.72 \%$ \\
\hline & 333333 & $2.24 \%$ \\
\hline & 996666 & $1.68 \%$ \\
\hline & 666666 & $1.60 \%$ \\
\hline & 999966 & $1.60 \%$ \\
\hline & cc9999 & $1.41 \%$ \\
\hline & ffcccc & $1.26 \%$ \\
\hline & 663333 & $1.21 \%$ \\
\hline & ccffff & $0.92 \%$ \\
\hline & ffffcc & $0.79 \%$ \\
\hline & $9999 \mathrm{cc}$ & $0.43 \%$ \\
\hline & 666699 & $0.38 \%$ \\
\hline & ffccff & $0.36 \%$ \\
\hline Total & 333300 & $0.29 \%$ \\
\hline & 669999 & $0.11 \%$ \\
\hline & 13 color & $1.15 \%$ \\
\hline & 33 color & $100.00 \%$ \\
\hline
\end{tabular}


color as dominant color and GY(22\%) of light grayish as assort color. The luxurious and classical image is expressed using PB of deep tone as accent color.

$<$ Table 19> Main color arrangement of LANCOME

\begin{tabular}{|c|c|c|}
\hline \multirow{3}{*}{$\begin{array}{c}\text { domina } \\
\text { nt } \\
\text { color }\end{array}$} & Hex & \#ffffff \\
\hline & Hue & 8.48GY 9.9/0.78 \\
\hline & RGB & $(255,255,255)$ \\
\hline \multirow{6}{*}{$\begin{array}{l}\text { assort } \\
\text { color }\end{array}$} & Hex & \#ebece7 \\
\hline & Hue & 7.5GY 9.15/1.09 \\
\hline & RGB & $(235,236,231)$ \\
\hline & Hex & $\# \mathrm{e} 0 \mathrm{e} 1 \mathrm{db}$ \\
\hline & Hue & 7.21GY 8.71/1.16 \\
\hline & RGB & $(224,225,219)$ \\
\hline \multirow{3}{*}{$\begin{array}{l}\text { accent } \\
\text { color }\end{array}$} & Hex & \#132179 \\
\hline & Hue & 6.71PB 1.25/12.58 \\
\hline & RGB & $(19,33,121)$ \\
\hline
\end{tabular}

9) Analysis of $\mathrm{O} \mathrm{HUI}$ color

The $\mathrm{O} \mathrm{HUl}$ web-site main page as $\langle$ Fig. 9$\rangle$ is divided in width 104 and height 65 with total 8,395 by Adobe Photoshop and the pixel transformed main page colors are totally 253. The similar colors showing the very low difference of $\mathrm{HV} / \mathrm{C}$ value are primarily classified

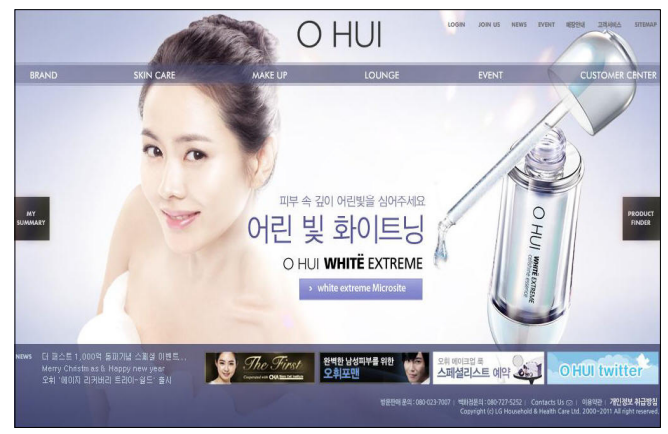

<Fig. 9> O HUl Homepage in 33 colors by color cache. Classifying the dominant color, assort color and accent color according to the distribution chart, the derived color combination is as <Table 21>. The calm, mild, gentle, natural, modest, gloomy image and composure are expressed using White of achromatic color as dominant color and PB of grayish and deep tone.

$<$ Table 20> O HUl color distribution chart

\begin{tabular}{c|c|c}
\hline \hline color & HTML Hex RGB & \multicolumn{1}{c}{ Ratio } \\
\hline & ffffff & $33.48 \%$ \\
\hline & ccccff & $12.23 \%$ \\
\hline & cccccc & $11.98 \%$ \\
\hline & ffcccc & $7.16 \%$ \\
\hline & 666699 & $5.83 \%$ \\
\hline & 666666 & $5.41 \%$ \\
\hline & 000000 & $4.94 \%$ \\
\hline & 999999 & $3.18 \%$ \\
\hline & $9999 \mathrm{cc}$ & $3.12 \%$ \\
\hline & 333333 & $2.49 \%$ \\
\hline & ffffcc & $2.23 \%$ \\
\hline & 336666 & $1.41 \%$ \\
\hline & $99 \mathrm{cccc}$ & $1.35 \%$ \\
\hline & ffccff & $0.68 \%$ \\
\hline & cc9999 & $0.58 \%$ \\
\hline & 333366 & $0.56 \%$ \\
\hline & cccc99 & $0.43 \%$ \\
\hline & ccffff & $0.43 \%$ \\
\hline & 996666 & $0.40 \%$ \\
\hline & 666633 & $0.18 \%$ \\
\hline & cc99cc & $0.13 \%$ \\
\hline etc. & 14 color & $1.80 \%$ \\
\hline Total & 35 color & $100.00 \%$ \\
\hline \hline
\end{tabular}

$<$ Table 21> Main color scheme of O HUI

\begin{tabular}{l|l|l|l|}
\hline \hline \multicolumn{1}{l|}{} & & \\
\hline
\end{tabular}


10) Analysis of SK-II color

The SK-II web-site main page as $\langle$ Fig. 10$\rangle$ is divided in width 128 and height 82 with total 8,395 by Adobe Photoshop and the pixel transformed main page colors are totally 256. The similar colors showing the very low difference of $\mathrm{HV} / \mathrm{C}$ value are primarily classified in 43 colors by color cache. Classifying the dominant color, assort color and accent color according to the distribution chart, the derived color combination is as <Table 23>. Two tones as dominant color(80\%) are used, the first YR and $R$ affiliation of deep tone express the deep, traditional, courteous, loyal, luxurious and classical image, and the second $Y$ and GY

<Table 22> SK-II color distribution chart

\begin{tabular}{|c|c|c|}
\hline color & HTML Hex RGB & Ratio \\
\hline & 660000 & $26.10 \%$ \\
\hline & ffffff & $21.65 \%$ \\
\hline & $\operatorname{cccccc}$ & $12.64 \%$ \\
\hline & ccccff & $4.13 \%$ \\
\hline & 000000 & $3.86 \%$ \\
\hline & $\mathrm{ffcccc}$ & $3.44 \%$ \\
\hline & ccffff & $3.39 \%$ \\
\hline & 990000 & $3.03 \%$ \\
\hline & 993333 & $2.72 \%$ \\
\hline & ffffcc & $2.65 \%$ \\
\hline & 330000 & $2.48 \%$ \\
\hline & 999999 & $2.32 \%$ \\
\hline & 99cccc & $1.87 \%$ \\
\hline & 996666 & $1.81 \%$ \\
\hline & cc9999 & $1.62 \%$ \\
\hline & 666666 & $1.12 \%$ \\
\hline & cc6666 & $0.89 \%$ \\
\hline & $\operatorname{cccc} 99$ & $0.67 \%$ \\
\hline & 669999 & $0.62 \%$ \\
\hline & 663333 & $0.50 \%$ \\
\hline & 663300 & $0.40 \%$ \\
\hline & 333333 & $0.15 \%$ \\
\hline & $99 \mathrm{ccff}$ & $0.10 \%$ \\
\hline etc. & 20 color & $1.90 \%$ \\
\hline Total & 43 color & $100.00 \%$ \\
\hline
\end{tabular}

affiliation of pale tone express the light, soft, weak, female image. The dull tone as dominant color expresses the calm, mild, gentle, dull, natural image and so the noble image.

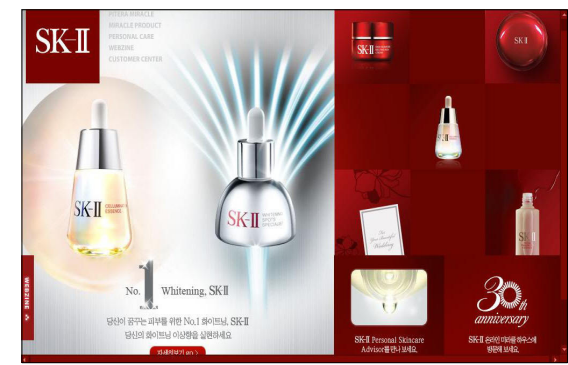

<Fig. 10> SK-II Homepage

$<$ Table 23> Main color arrangement of SK-II

\begin{tabular}{l|l|l|l|}
\hline & & \\
\hline
\end{tabular}

\section{Image analysis of web-site color}

<Fig. 11> shows the color arrangement image and the color arrangement according to the color tome analysis are positioned on $\mid \mathrm{RI}$ color 
arrangement image scale, and <Fig. 12> derived the emotional languages in accordance with adjective image scale.

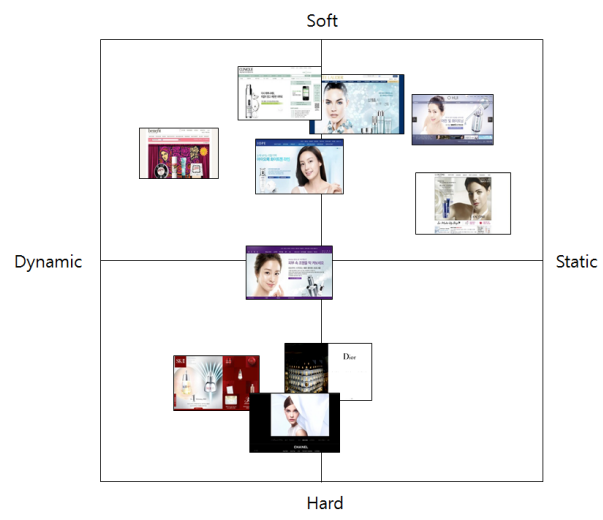

<Fig. 11> Distribution of web-site color scheme

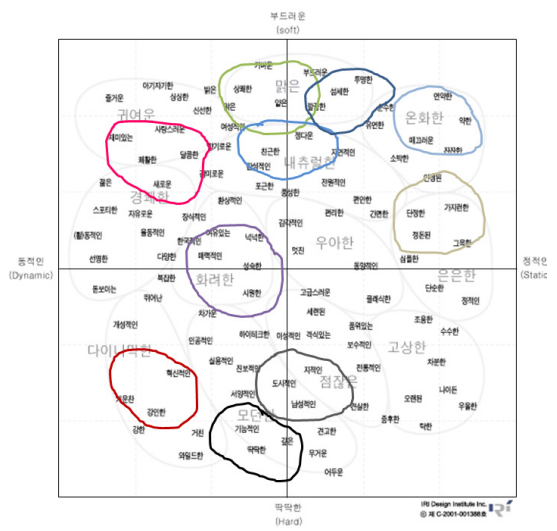

<Fig. 12> Adjective image of web-site
The color analysis results of 10 cosmetic web-sites show that all web-site main pages used White and White affiliation as dominant color, however the distribution of Black affiliation is large at CHANEL and Dior, and Green affiliation is largely distributed at CLINIQUE and LANCOME. IOPE and ESTEE LAUDER used Blue affiliation, $\mathrm{O} \mathrm{HUI}$ and HERA used Purple affiliation, benefit and SK- II sued Red affiliation as dominant color respectively. Based on these results, the cosmetic brands are classified as 5 groups for analysis of color tone image. The survey is empirically analyzed substituting the color tone image of pre studies and the emotional languages of $|R|$ sensitive image scale.

\section{Image analysis of affiliated color}

Regarding the color association of 5 color groups, the survey is empirically analyzed based on the adjectives of pre studies and the adjectives applied to $|\mathrm{R}|$ image scale and pre studies as <Fig. 13>. The results are as follows.

At the question on the feeling of Black\&White, luxurious 127 people(39\%) is followed by modern 84 people(26\%), polished 68 (21\%) and gentle $46(14 \%)$. The harmony between the calming

$<$ Table 24> Emotional language of color affiliation

\begin{tabular}{c|c|c|c}
\hline \hline \multirow{2}{*}{ Affiliation } & \multirow{2}{*}{ Brand } & \multicolumn{2}{|c}{ Emotional language } \\
\cline { 2 - 4 } & CHANEL & modern & Pre studies \\
\hline Black & Dior & gentle & luxurious \\
\&White & CLINIQUE & clean \\
\hline Green & LANCOME & quiet & stable \\
\&White & IOPE & natural & pure \\
\hline Blue & ESTEE LAUDER & naive & cool \\
\&White & O HUI & splendid & mysterious \\
\hline Purple & HERA & moderate & elegant \\
\&White & benefit & cute & voluptuous \\
\hline Red & SK-II & dynamic & passionate \\
\hline \hline
\end{tabular}


effect of Black, and the innocence and honest effect of White is well matching to the color arrangement due to the luxurious and simple character of the color, and the modern image is shown distinguishing the design shape and the lines.
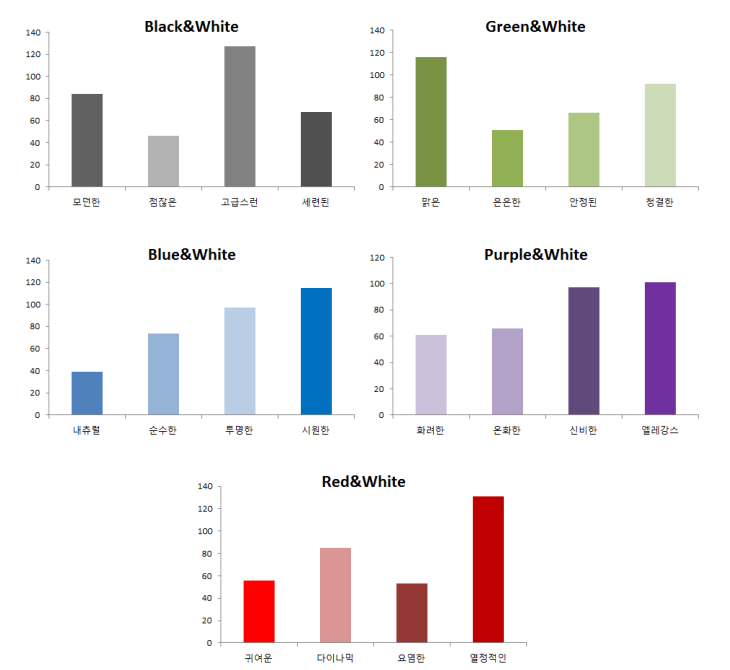

<Fig. 13> Survey results on web-site adjective image

At the question on Green\&White, clean 116 people $(36 \%)$ is followed by pure 92 people(28\%), stable $66(20 \%)$ and quiet $51(16 \%)$. The clean feeling Green and White commonly share, and the pure image of White by itself are well delivered to consumers. The color arrangement of Tone on Tone seems to give the moderate and quiet feeling.

At the question on Blue\&White, cool 115 people(35\%) is followed by transparent 97 people $(30 \%)$, naive $74(23 \%)$ and natural $39(12 \%)$. The combination of White and chroma low Blue makes a cool feeling, and it is effective to arrange the colors with White in order to express the transparent mood. It is analogically interpreted that the naive and natural feeling is resulted from the concrete association such as heaven and sea.

Regarding Purple\&White, elegant $101(31 \%)$ is followed by mysterious 97(30\%), moderate 66(20\%), splendid 61(19\%). The affiliated color of chroma low Purple is used for representation of elegance, and the mystery seems to be resulted from the effective color arrangement of White with low brightness. The chroma low color arrangement gives the moderate feeling, and the splendid image of Purple is used by itself.

Regarding Red\&White, passionate 131 people $(40 \%)$ is followed by dynamic 85 people(26\%), cute 56 people(17\%), voluptuous 53 people(17\%). The passionate and dynamic image are strongly expressed because of the high attractiveness of color, Red. The cute image and voluptuous feeling seem to be resulted from color arrangement. Chroma high and brightness low Red and White are able to give the cute image, and the voluptuous feeling probably comes from Red rather than unmixed color supported by achromatic color.

\section{Conclusion}

This study derived the color trend of each cosmetic brand and emotional images analyzing the main page color of cosmetic web-site, and investigated the influence of the web-site color tone and color tone image as the information transferring visual factor on the effective communication.

According to the study results, all brands used the White affiliation as base, and each image is expressed arranging Black, Red, Green and Purple as dominant color or assort color in order to deliver the brand and product image. It is analyzed that the most web-sites are using 
the pale and grayish tone as color tone in order to express the female, soft, clean, elegant and polished image.

The analysis results about the influence of cosmetic web-site color image are summarized as follows

Regarding Black \& White affiliation, luxurious and modern image are answered as majority. Therefore, it will be effective to apply them to the masterpiece or luxurious brand aiming image. They will be also effective to maximize the brand image through the unique brightness contrast of the achromatic color possessing the visual recognitive effects.

Regarding Green\&White affiliation, clean and pure image are answered as majority. It will be effective to apply to the freshness and cleanness emphasizing products. They are also matching to the color image for the basic and ant aging products emphasizing the cleanness and purity.

Regarding Blue\&White affiliation, the cool and transparent image are majority and the chroma high Blue color is arranged in order that web-site of the basic and functional product is matching to the product purpose. Thus, the cool and transparent image are effectively expressed.

Regarding Purple\&White affiliation, the answer "elegant and mysterious' is a little high, but the differences are not large. Thus, the delivery of the various images is desired through the effective color arrangement rather than the main color tone.

Regarding Red\&White affiliation, the answer "passionate and dynamic" is majority. The passionate and dynamic image of Red affiliation will be a strong tool to change the established image or promote the new business.

Regarding the additional question on the most ideal color tone of cosmetic brand web-site,
46.8\% answered the achromatic color affiliation(White, Black, Grey), whereas 19.7\% answered the Red affiliation. Therefore, The brand awareness is considered to be significantly correlated to the color preference.

This study excluded the color external factors on web-site and limited only colors, and the survey is finished also in limited areas. Therefore, the study results hardly will be generalized. However, the survey's responses are not largely different to the scope of theoretically established color association image of many pre studies, and the image preference of color tone is relatively obvious.

The coming studies should extend the survey sample size for the generalization and continuously proceeded to investigate the detailed results like the successful color marketing cases of web color image and the sequential sale volume.

This study results are necessary for plan of color delivering the strong transferring power of visual information the time when we make the effective web-sites, and it hopefully will be utilized as the basic data for the color marketing to actively express the brand identity and to satisfy the consumers' sensitivity.

\section{Reference}

1) Liu, Yuan-Chen (2010), "A Study On Color Application of Brand BI and Web Site", Dept. of Design Graduate School of Design \& IT Dongseo University, pp.18-19.

2) Park, Yeon Sun (2007), Color, Yerim. p.6.

3) TRULY DEEPLY (2010), "2009/2010 Trend Report - The visual Language of Brand", pp.140-141.

4) Park, Sook Hee, Jung, Hyang Jin (2010), 
Basic Color \& Design, Taehakwon, p.112.

5) Kim, Ju-Hoon (2007), " A Study on the Brand Color Application Affecting Brand Image", Journal of Korean Society of Communication Design, 10, p.45.

6) Kim, Mee Shuk (2003), "A study on Color analysis for web design - focused on the on-line education site", Korean Journal of Communication Studies, 13, pp.78-79.

7) Kim, Kyung-Hwa, Na Ji-Young (2011), "Color Marketing Strategy of Milk Packaging", The Journal of the Korea Contents Association, 12(1), p.198.

8) Kim, Shin-Ae, Choi, Ho-Gyu, Kwon, Ki-Dae (2012), “A Relational Study among Color Marketing Strategy, Brand Charisma and Customer's Brand Satisfaction - Focused on Goodtrae Co-brand", Korean Journal of Food Marketing Economics, 29(1), p.39.

9) Faber Biren (1993), Psychology of Color, trans, Kim, Hwa Jung, Dongkuk publishing company, pp.318-321.

10) Moon, Ji-Young, Kim, Ji-Yeon, Cho, Ju-Yeon, Lee, Kyu-Hye (2011), "A Study of Color Characteristics of the Websites of Sportswear Brands", The Research Journal of the Costume Culture, 19(4), pp.796-798.

11) Kim, Hyun Jeong (2010), "Evaluation of Color Application of Homepage of Domestic and Foreign Online bookstores - Focused on the Quantitative Evaluation of Aesthetic Measure and Cross-national Comparative Analysis", Journal of Korea Design Knowledge, 10(4).
12) Brown, David C, Esteban Burbano, Minski (2002), "Evaluating Web Page Color and Layout Adaptation." IEEE Mutimedia, 9(1).

13) Bernd Schmitt, Alex Simonson (1997), "Marketing Aesthetics-The Strategy of Brands", The Free Press.

14) Moon, Parry Spencer, Domina Eberle (1944), "Aesthetic Measure Applied to Color Harmony", Journal of the Optical Society of America. 34(4)

15) W. O. Bearden, T. A. Shimp (1982), "The Use of Extrinsic Cues to Facilitate Product Adaptation," Journal of Marketing Research, 18(1).

16) R. W. Belk (1975), "Situation Variables in Consumer Behavior," Journal of Consumer Research, 2(12).

17) Igarashi Haruyoshi, Hamada Setsuo (1998), "The Effect of i.c.v. Administration of Calcitionin on Mouse Small Intestinal Transit," The Japnese Journal of pharmacology.

18) R. J. Kent, C. T. Allen (1994), "Competitive Inference Effects in Consumer Memory for Advertising: The Role of Brand Familiarity", Journal of Marketing, 58(1).

접수일(2012년 11월 26일),

수정일(1차 : 2012년 12월 11일),

게재확정일(2012년 12월 18일) 